\title{
Hearing Screening Combined with Target Gene Panel Testing Increased Etiological Diagnostic Yield in Deaf Children
}

\author{
Le Xie, Yue Qiu (D), Yuan Jin, Kai Xu, Xue Bai, Xiao-Zhou Liu, Xiao-Hui Wang, Sen Chen (D), \\ and Yu Sun
}

Department of Otorhinolaryngology, Union Hospital, Tongji Medical College, Huazhong University of Science and Technology, Wuhan 430022, China

Correspondence should be addressed to Sen Chen; senchen@hust.edu.cn and Yu Sun; sunyu@hust.edu.cn

Received 21 April 2021; Revised 9 June 2021; Accepted 11 July 2021; Published 23 July 2021

Academic Editor: Geng lin $\mathrm{Li}$

Copyright ( 2021 Le Xie et al. This is an open access article distributed under the Creative Commons Attribution License, which permits unrestricted use, distribution, and reproduction in any medium, provided the original work is properly cited.

\begin{abstract}
Genetic testing is the gold standard for exploring the etiology of congenital hearing loss. Here, we enrolled 137 Chinese patients with congenital hearing loss to describe the molecular epidemiology by using 127 gene panel testing or 159 variant testing. Sixty-three deaf children received 127 gene panel testing, while seventy-four patients received 159 variant testing. By use of 127 gene panel testing, more mutant genes and variants were identified. The most frequent mutant genes were GJB2, SLC26A4, MYO15A, CDH23, and OTOF. By analyzing the patients who received 127 gene panel testing, we found that 51 deaf children carried variants which were not included in 159 variant testing. Therefore, a large number of patients would be misdiagnosed if only 159 variant testing is used. This study highlights the advantage of 127 gene panel testing, and it suggests that broader genetic testing should be done to identify the genetic etiology of congenital hearing loss.
\end{abstract}

\section{Introduction}

Congenital hearing loss (HL) is a common disease, and about 1 to 2 per 1000 live births suffer from congenital hearing loss in the world. The prevalence of hearing loss increases with age. Among the kids in the primary school, the prevalence is $2.83 / 1000$. Furthermore, in adolescents, it will rise to $3.5 / 1000[1-3]$. According to the latest date, approximately $50 \%-60 \%$ of congenital HL is caused by genetic factors $[4,5]$. However, genetic disorder can also cause late-onset deafness in children and adolescents [2]. Both congenital or late-onset deafness caused by genetic disorder can be attributed to hereditary hearing loss. To date, more than 150 deafness-related genes and 6000 variants have been identified [6] (https://hereditaryhearingloss.org). The hearing loss genes and hot variants showed distinctly in different ethnic groups. Among these hearing loss genes, GJB2 and SLC26A4 gene variants were most common in Chinese deaf population [4]. For GJB2 gene variants, the most frequent etiological factor of nonsyndromic hereditary hearing loss, the hotspots were described as c.235delC and c.109G $>$ A, common in Asian, c.35delG, found in European, and c.71G $>A$, predominant in the Indian [7, 8]. Although GJB2 and SLC26A4 mutations account for a large part of causes of hereditary deafness, there are still a large number of known or unknown gene mutations that cause hearing loss. How to quickly and accurately detect causes of patients with suspected hereditary deafness is an important issue in the diagnosis and treatment of deafness.

Genetic testing is the gold standard for exploring the etiology of congenital and late-onset deafness. In addition, medical history and other auditory physiology tests are also very important. For hereditary deafness, genetic testing is a crucial step. Except for finding causes, it also could help diagnosis and intervention of syndromic hearing loss because some children only exhibited hearing loss without other symptoms at a young age. Besides, genetic testing was beneficial to protecting children with some variants such as m.1555A > G or variants in KCNQ1 gene from avoidable risk factors [9-12]. DNA sequencing first 
TABLE 1: The basic information of patients recruited in this research.

\begin{tabular}{lccc}
\hline & 127 gene panel & 159 variants & Total \\
\hline Male & 35 & 39 & 74 \\
Female & 28 & 35 & 63 \\
Total & 63 & 74 & 137 \\
\hline
\end{tabular}

TABLE 2: The genetic spectrum of hereditary hearing loss patients detected by 127 gene panel testing.

\begin{tabular}{|c|c|c|c|c|}
\hline Gene & $\begin{array}{c}\text { Patients } \\
\text { carried variant }\end{array}$ & $\begin{array}{c}\text { Pathogenic } \\
\text { variant }\end{array}$ & $\begin{array}{c}\text { Likely } \\
\text { pathogenic } \\
\text { variant }\end{array}$ & $\begin{array}{c}\text { VUS } \\
\text { variant }\end{array}$ \\
\hline GJB2 & 33 & 32 & 0 & 1 \\
\hline SLC26A4 & 16 & 15 & 0 & 1 \\
\hline OTOF & 5 & 1 & 3 & 1 \\
\hline MYO15A & 5 & 1 & 1 & 3 \\
\hline $\mathrm{CDH} 23$ & 5 & 0 & 2 & 3 \\
\hline TECTA & 4 & 0 & 1 & 3 \\
\hline MYO7A & 4 & 0 & 0 & 4 \\
\hline WFS1 & 3 & 1 & 0 & 2 \\
\hline PTPRQ & 2 & 1 & 0 & 1 \\
\hline $\mathrm{COCH}$ & 2 & 0 & 2 & 0 \\
\hline GJB3 & 2 & 0 & 1 & 1 \\
\hline OTOA & 2 & 0 & 1 & 1 \\
\hline USH $2 A$ & 2 & 0 & 1 & 1 \\
\hline ESPN & 2 & 0 & 0 & 2 \\
\hline TRIOBP & 2 & 0 & 0 & 2 \\
\hline GRXCR1 & 1 & 1 & 0 & 0 \\
\hline ALMS1 & 1 & 1 & 0 & 0 \\
\hline COL4A3 & 1 & 0 & 1 & 0 \\
\hline ILDR 1 & 1 & 0 & 1 & 0 \\
\hline $\begin{array}{l}A D G R \\
V 1\end{array}$ & 1 & 0 & 0 & 1 \\
\hline CLDN14 & 1 & 0 & 0 & 1 \\
\hline CRYM & 1 & 0 & 0 & 1 \\
\hline DIAPH3 & 1 & 0 & 0 & 1 \\
\hline EDNRB & 1 & 0 & 0 & 1 \\
\hline LOXHD1 & 1 & 0 & 0 & 1 \\
\hline MITF & 1 & 0 & 0 & 1 \\
\hline МYH14 & 1 & 0 & 0 & 1 \\
\hline PCDH15 & 1 & 0 & 0 & 1 \\
\hline PDSS1 & 1 & 0 & 0 & 1 \\
\hline SLC17A8 & 1 & 0 & 0 & 1 \\
\hline TJP2 & 1 & 0 & 0 & 1 \\
\hline TMC1 & 1 & 0 & 0 & 1 \\
\hline
\end{tabular}

described 44 years ago has evolved from single-mutation sequencing (Sanger sequencing) to high-throughput sequencing (Next generation sequencing, NGS) [5, 13]. Compared to Sanger sequencing, NGS was able to simultaneously sequence millions of small fragments at a reasonable cost and reduced
TABLE 3: The genetic spectrum of hereditary hearing loss patients detected by 159 variant testing.

\begin{tabular}{lcc}
\hline Gene & Patients with mutant gene & Percentage \\
\hline GJB2 & 45 & 0.608108 \\
SLC26A4 & 27 & 0.364865 \\
GJB3 & 2 & 0.027027 \\
MT-RNR1 & 1 & 0.013514 \\
\hline
\end{tabular}

runtime [13]. Targeted gene panel, a common NGS technique, has been widely applied in deaf population. It was targeted to the detection of variants in the massively known hearing loss genes. Considering the cost and time consuming, different panels were designed in clinical applications $[1,4,12,14,15]$.

As the spectrum of gene mutations is distinct in different ethnic group, studying mutated character of Chinese deaf children and choosing the appropriate gene panel are critical for clinical diagnosis and treatment. In this study, we enrolled 137 Chinese children with sensorineural hearing loss to describe the molecular epidemiology by using 127 gene panel testing and 159 variants in 22 gene testing.

\section{Materials and Methods}

2.1. Patients and Samples. A total of 137 patients under the age of twelve with sensorineural hearing loss were recruited from the Wuhan Union Hospital from 2018 to 2020. All patients failed pass the neonatal hearing screening and underwent 127 gene panel testing (targeting the exon regions and exon-intron boundaries of 127 known deafness-causing nuclear genes as well as deafness-causing mitochondrial regions) or 159 variants in 22 gene testing (detecting a total of 159 hotspot variants in 22 known deafness-causing genes). Informed consent was obtained from all patients or their legal guardians. This study was approved by the Institutional Review Board of the BGI in accordance with the Declaration of Helsinki (1964). Written informed consent, clinical evaluations, and blood samples were obtained from all the participants or their legal guardians. The study of the protocols was approved by the review boards of the ethics committees of the Tongji Medical College of Huazhong University of Science and Technology.

Peripheral venous blood samples were collected from all recruited patients. Genomic DNA was obtained and purified using a QIAamp DSP DNA Blood Mini Kit (61104, Qiagen Inc., Valencia, CA, USA).

2.2. Library Preparation, Sequencing, and Bioinformatics. Fragmented DNA (a size of 350-400 base pairs) were prepared using Covaris LE220 ultrasonicator (Covaris Inc., Woburn, Massachusetts, USA). Then end-repair and adaptor ligation were performed for library construction. After array hybridization, elution, and postcapture amplification, targeted DNA fragments were sequenced on the BGISEQ500 platform. After sequencing and quality control, the clean reads derived from targeted high-throughput sequencing 


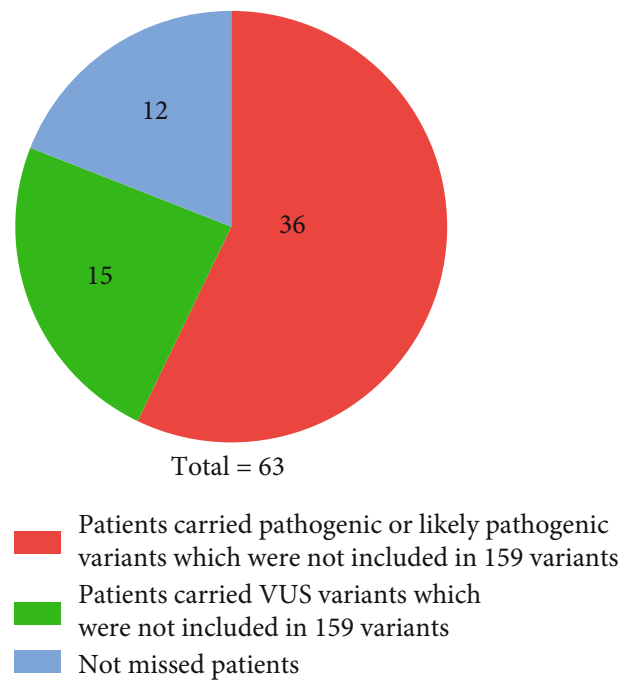

(a)

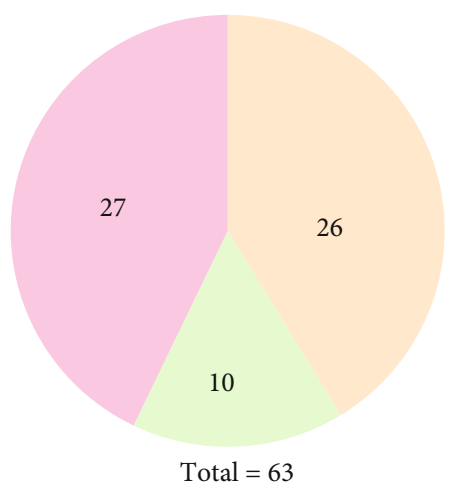

Misdiagnosed patients carried pathogenic or likely pathogenic variants

Missed diagnosis patients carried VUS variants

Not missed diagnosed patients

(b)

FIgURe 1: Analyze the genetic etiology of patients who received 127 gene panel testing compared with 159 variants. (a) 36 patients carried pathogenic or likely pathogenic variants which were not included in 159 variants, fifteen patients carried VUS variants which were not included in 159 variants, and 12 patients do not carry the variants which were not included in 159 variants. (b) 26 patients who carried pathogenic or likely pathogenic variants would misdiagnose if they were only detected by 159 variant testing, ten patients who carried VUS might miss diagnosis, and twenty-seven patients would not miss diagnosis.

were aligned to the GRCh37/hg19 using the BWA (Burrows Wheeler Aligner) Multi-Vision software package. After alignment, the variants of single-nucleotide variants (SNVs) and inserts and deletions (InDels) were detected by GATK software. Then, filtered SNVs and InDels were compared with NCBI GenBank database, 1000 Genomes, ESP6500, dbSNP, HGMD, and ExAC. Candidate variants were classified into pathogenic variants, likely pathogenic variants, variants of uncertain significance (VUS), likely benign variants, and benign variants according to the American College of Medical Genetics and Genomics-Association for Molecular Pathology (ACMG-AMP) guideline. The method described in this study partly reproduces the wording in our previous article [16-18].

\section{Result}

3.1. Study Samples. A total of 137 patients were included in this research, seventy-four patients were male and sixtythree were female. Sixty-three patients (46\%) received genetic testing by 127 gene panel, while seventy-four patients (54\%) received 159 variant testing (Table 1 ). Tested genes are provided in supplement (Table S1 and S2.)

3.2. Genetic Spectrum. Among the 63 received 127 gene panel test, ninety-two variants in 32 genes were detected, the top five genes were GJB2 (33 patients), SLC26A4 (16 patients), MYO15A (5 patients), CDH23 (5 patients), and OTOF (5 patients) (Table 2).

Among the 74 who received 159 variant testing, twentyone variants in 4 genes were detected; the patients with mutation of GJB2, SLC26A4, GJB3, and MT-RNR1 were 45, 27, 2, and 1 , respectively (Table 3 ).
3.3. Different Diagnosis between 127 Gene Panel Testing and 159 Variant Testing. Among the 63 patients who received 127 gene testing, fifty-one patients were found to carry variants which were not included in 159 variant testing, thirtysix of them had been found to carry pathogenic or likely pathogenic variants. If these patients received 159 variant testing only, in 36 of them, the genetic etiology of the hearing loss would be missed, and 26 of the patients were found to carry the pathogenic or likely pathogenic variants (Figure 1). Twenty-two deafness gene mutation which was not included in 159 variant testing was found in 25 patients; nine of them carried pathogenic or likely pathogenic variants of these 22 deafness genes. In these 22 deafness genes which were not included in 159 variant testing, eighty-four variants were found, ten of them were pathogenic or likely pathogenic (Table 4).

3.4. GJB2 Variants. In 127 gene panel testing, six variants of GJB2 were identified; c.109G>A, c.235delC, c.299_300del AT, c.176_191del GCTGCAAGAACGTGTG, c.139G>T, and $c .88 \mathrm{~A}>\mathrm{G}$ were detected $18,15,5,2,1$, and 1 times, respectively (Table 5 ). The patients who carried heterozygous variant of c.109G $>$ A were the most, and secondary was heterozygous c.235delC mutation in 11 patients, and then was the homozygous c.109G $>$ A mutation in 5 patients and homozygous c.235delC mutation in 4 patients. In the 11 patients who carried heterozygous c.235delC variants, four of them carried c.299_300del AT, 2 carried c.109G $>$ A, and 2 carried c.176_191del GCTGCAAG AACGTGTG compound heterozygotes variants, respectively. In our study, a patient who carried a novel heterozygous variant was identified, the nucleotide change is $c .88 \mathrm{~A}>\mathrm{G}$, the protein change is p.Ile $30 \mathrm{Val}$, and the characterization of variant 
TABLE 4: The variants would be missed if tested by 159 variant testing.

\begin{tabular}{|c|c|c|c|}
\hline Gene & Variant & Effect on protein & Characterization of variant \\
\hline \multirow{2}{*}{$A D G R V 1$} & c. $11704 \mathrm{~A}>\mathrm{G}$ & p.Met3902Val & VUS \\
\hline & c. $2899-10 \mathrm{~T}>\mathrm{A}$ & & VUS \\
\hline \multirow[t]{4}{*}{ ALMS1 } & c. $2035 \mathrm{C}>\mathrm{T}$ & p.Arg679Ter & Pathogenic \\
\hline & c. $5067+1 \mathrm{G}>\mathrm{A}$ & & Likely pathogenic \\
\hline & c. $6604 \mathrm{G}>\mathrm{A}$ & p.Asp2202Asn & Likely pathogenic \\
\hline & c. $1765 \mathrm{G}>\mathrm{A}$ & p.Asp589Asn & VUS \\
\hline \multirow{5}{*}{$\mathrm{CDH} 23$} & c. $2368 \mathrm{~A}>\mathrm{G}$ & p.Met790Val & VUS \\
\hline & c. $3262 \mathrm{G}>\mathrm{A}$ & p.Val1088Met & VUS \\
\hline & c. $4859 \mathrm{~T}>\mathrm{A}$ & p.Val1620Glu & VUS \\
\hline & c. $5051 \mathrm{G}>\mathrm{A}$ & p.Arg1684His & VUS \\
\hline & c. $805 \mathrm{C}>\mathrm{T}$ & p.Arg269Trp & VUS \\
\hline \multirow{2}{*}{ CLDN14 } & c. $449 \mathrm{C}>\mathrm{T}$ & p.Pro150Leu & VUS \\
\hline & c. $694 \mathrm{G}>\mathrm{A}$ & p.Gly232Arg & VUS \\
\hline $\mathrm{COCH}$ & c.812_813insG & p.Val271Valfs X5 & Likely pathogenic \\
\hline COL4A3 & c. $4755+1 \mathrm{G}>\mathrm{A}$ & & Likely pathogenic \\
\hline$C R Y M$ & c. $849 \mathrm{C}>\mathrm{T}$ & p.His 283 His & VUS \\
\hline DIAPH3 & c.3543_3544i nsC & p.Pro1181 Profs $* 4$ & VUS \\
\hline$E D N R B$ & c. $758 \mathrm{G}>\mathrm{A}$ & p.Arg253Gln & VUS \\
\hline \multirow{2}{*}{$E S P N$} & c. $1828 \mathrm{G}>\mathrm{C}$ & p.Ala610Pro & VUS \\
\hline & c. $2069 \mathrm{C}>\mathrm{T}$ & p.Ser690Leu & VUS \\
\hline \multirow{3}{*}{ GJB2 } & c. $109 \mathrm{G}>\mathrm{A}$ & p.Val37Ile & Pathogenic \\
\hline & c. $139 \mathrm{G}>\mathrm{T}$ & p.Glu47Ter & Pathogenic \\
\hline & c. $88 \mathrm{~A}>\mathrm{G}$ & p.Ile30Val & VUS \\
\hline \multirow{2}{*}{ GJB3 } & c. $580 \mathrm{G}>\mathrm{A}$ & p.Ala194Thr & Likely pathogenic \\
\hline & c. $595 \mathrm{~A}>\mathrm{G}$ & p.Ile199Val & VUS \\
\hline GRXCR1 & c. $784 \mathrm{C}>\mathrm{T}$ & p.Arg262Ter & Pathogenic \\
\hline ILDR1 & c. $206 \mathrm{C}>\mathrm{A}$ & p.Pro69His & Likely pathogenic \\
\hline LOXHD1 & EX31 DUP & & VUS \\
\hline MITF & c. $950 \mathrm{G}>\mathrm{C}$ & p.Arg317Thr & VUS \\
\hline \multirow[t]{4}{*}{ MYH14 } & c. $4641 \mathrm{C}>\mathrm{T}$ & p.Asp1547Asp & VUS \\
\hline & c.10251_10253 delCTT & & Pathogenic \\
\hline & c. $4642 \mathrm{G}>\mathrm{A}$ & p.Ala1548Thr & Pathogenic \\
\hline & c.8702_8703i nsT & p.Pro2901Pro fsX25 & Likely pathogenic \\
\hline \multirow[t]{5}{*}{ MYO15A } & c. $3239 \mathrm{G}>\mathrm{A}$ & p.Arg1080His & VUS \\
\hline & c. $3743 \mathrm{G}>\mathrm{A}$ & p.Arg1248Gln & VUS \\
\hline & c. $5681 \mathrm{~T}>\mathrm{C}$ & p.Leu1894Pro & VUS \\
\hline & c. $6340 \mathrm{G}>\mathrm{A}$ & p.Val2114Met & VUS \\
\hline & c. $1901 \mathrm{G}>\mathrm{A}$ & p.Arg634Gln & VUS \\
\hline \multirow{4}{*}{ MYO7A } & c. $1945 \mathrm{C}>\mathrm{T}$ & p.Arg649Trp & VUS \\
\hline & c. $6235 \mathrm{C}>\mathrm{T}$ & p.Arg2079Trp & VUS \\
\hline & c. $6470 \mathrm{~T}>\mathrm{C}$ & p.Ile2157Thr & VUS \\
\hline & c.943delT & p.Ser315Profs $* 5$ & Likely pathogenic \\
\hline \multirow[t]{4}{*}{ OTOA } & c. $1172 \mathrm{C}>\mathrm{T}$ & p.Ser391Leu & VUS \\
\hline & c. $2359 \mathrm{G}>\mathrm{T}$ & p.Glu787Ter & VUS \\
\hline & c. $5816 \mathrm{G}>\mathrm{A}$ & p.Arg1939Gln & Pathogenic \\
\hline & c. $4023+1 \mathrm{G}>\mathrm{A}$ & & Likely pathogenic \\
\hline \multirow[t]{3}{*}{ OTOF } & c. $5098 \mathrm{G}>\mathrm{C}$ & p.Glu1700Gln & Likely pathogenic \\
\hline & c. $1194 \mathrm{~T}>\mathrm{A}$ & p.Asp398Glu & VUS \\
\hline & c. $3429 \mathrm{G}>\mathrm{A}$ & p.Arg1143Arg & VUS \\
\hline
\end{tabular}


TABLE 4: Continued.

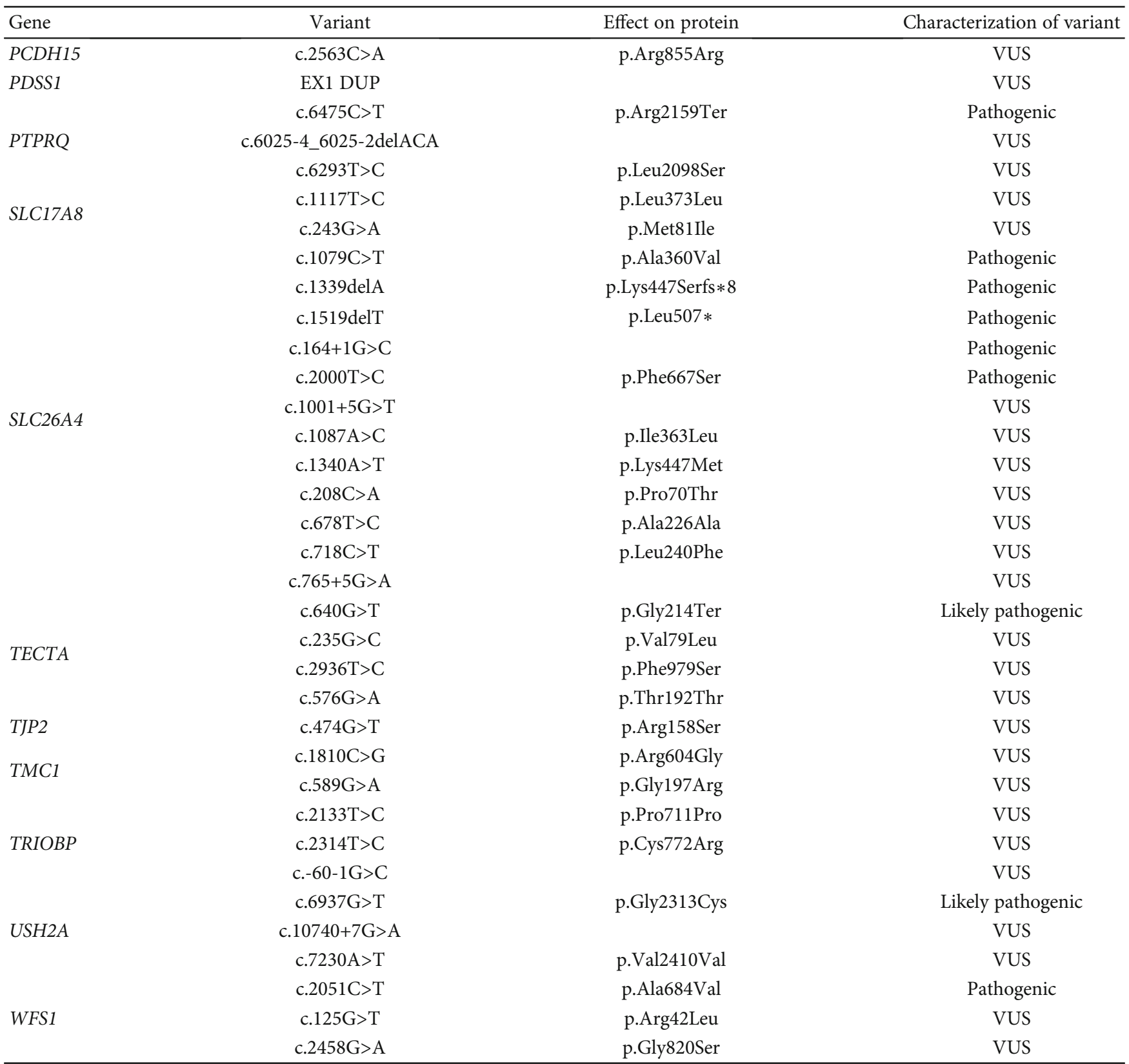

is VUS; this patient carried c.919-2A $>\mathrm{G}$ and c.1229C $>\mathrm{T}$ compound heterozygotes variants of SLC26A4 gene as well.

In 159 variant testing, eight variants of GJB2 were identified; the frequency of these variants from high to low were c.235delC, c.299_300delAT, c.176_191del GCTGCAAGA ACGTGTG, с.139G $>$ T, с. $187 \mathrm{G}>\mathrm{T}$, с. $257 \mathrm{C}>\mathrm{G}$, c. $427 \mathrm{C}>\mathrm{T}$, and c.428G $>\mathrm{A}$; the check-out times of these variants were $36,9,5,2,1,1,1$, and 1 , respectively. Nineteen patients carried heterozygous c.235delC variants, five of them carried c.299_300delAT compound heterozygotes variants, three patients carried c.176_191del GCTGCAAGAACGTGTG compound heterozygotes variants, and one carried
c.257C>G compound heterozygotes variants. The homozygous c.235delC patients were seventeen. c.299_300delAT heterozygous variant was identified in 9 patients, two of them carried c.176_191delGCTGCAAGAACGTGTG compound heterozygotes variants.

3.5. SLC26A4 Variants. In 127 gene panel testing, seventeen variants were identified in SLC26A4 gene, the most frequent variant detected was c.919-2A $>\mathrm{G}$, eleven patients carried heterozygous c.919-2A $>\mathrm{G}$ variant, nine of them were compound heterozygotes variants with other SLC26A4 variants, and one homozygous c.919-2A>G was identified. Eight novel variants were identified in our research; c.1339delA, 
TABLE 5: Variants frequency in GJB2 gene.

\begin{tabular}{lccc}
\hline Variants & Homozygous & Heterozygous & Total \\
\hline Testing by 127 gene panel & 5 & 13 & 18 \\
c.109G $>$ A & 4 & 11 & 15 \\
c.235delC & 1 & 4 & 5 \\
c.299_300del AT & 0 & 2 & 2 \\
c.176_191del GCTGCAAG & 1 & 0 & 1 \\
AACGTGTG & 0 & 1 & 1 \\
c.139G $>$ T & & & \\
c.88A $>$ G & 17 & 19 & 36 \\
Testing by 159 variant testing & 0 & 9 & 9 \\
c.235delC & 0 & 5 & 5 \\
c.299_300delAT & & & \\
c.176_191delGCTGCAAGA & 0 & 0 & 2 \\
ACGTGTG & 0 & 1 & 1 \\
c.139G $>$ T & 0 & 0 & 1 \\
c.187G $>$ T & 1 & 1 & 1 \\
c.427C $>$ T & 0 & 1 & 1 \\
c. $428 G>$ A & 0 &
\end{tabular}

c.1519delT, c.164+1G>C, and c.2000T $>C$ were likely pathogenic variants, while c. $1340 \mathrm{~A}>\mathrm{T}, \mathrm{c} .718 \mathrm{C}>\mathrm{T}, \mathrm{c} .765+5 \mathrm{G}>\mathrm{A}$, and c.208C $>$ A were VUS variants (Table 6).

Twelve variants of SLC26A4 were identified in 159 variant testing; the most common variant was c.919-2A $>\mathrm{G}$ same as the 127 gene panel testing, then was c.1229C>T and c.2168A $>$ G. Nineteen patients carried c.919-2A>G variant, fourteen of them were heterozygous while 5 were homozygous.

\section{Discussion}

Hearing scanning combined with molecular genetic testing is an effective approach to diagnose hereditary hearing loss, while because of the high cost of genetic testing, such limited the usage of more comprehensive genetic testing. Through 127 gene panel testing, more genetic etiology of deaf children was identified. In 127 gene panel testing, thirty-two deafness genes were identified in patients. The five most frequent genes were GJB2 (33 patients), SLC26A4 (16 patients), MYO15A (5 patients), OTOF (5 patients), and CDH23 (5 patients), while only four deafness genes were identified by 159 variant testing, and the frequency of these four genes was 45 (GJB2), 27 (SLC26A4), 2 (GJB3), and 1 (MT-RNR1), respectively. The genetic spectrum of 127 gene testing is consistent with the previous research in China [19]. The diagnostic rate of GJB2 and SLC26A4 is higher in 159 variant testing which may cause more mutant genes identified in 127 gene panel testing. Furthermore, eightyfour variants in 32 genes which were not included in 159 variant testing were identified, and ten variants of these were pathogenic or likely pathogenic. This result demonstrates that even though 159 variant testing contain hotspot deafness variants, it would still misdetect abundant of gene mutations.
TABLE 6: Variants frequency in SLC26A4 gene.

\begin{tabular}{|c|c|c|c|}
\hline Variants & Homozygous & Heterozygous & Total \\
\hline \multicolumn{4}{|c|}{ Testing by 127 gene panel } \\
\hline c. $919-2 A>G$ & 1 & 11 & 12 \\
\hline c. $2000 \mathrm{~T}>\mathrm{C}$ & 0 & 2 & 2 \\
\hline c. $1087 \mathrm{~A}>\mathrm{C}$ & 0 & 1 & 1 \\
\hline c. $1174 \mathrm{~A}>\mathrm{T}$ & 0 & 1 & 1 \\
\hline c.1339delA & 0 & 1 & 1 \\
\hline c. $164+1 \mathrm{G}>\mathrm{C}$ & 0 & 1 & 1 \\
\hline c. $718 \mathrm{C}>\mathrm{T}$ & 0 & 1 & 1 \\
\hline c. $1226 \mathrm{G}>\mathrm{A}$ & 0 & 1 & 1 \\
\hline c. $678 \mathrm{~T}>\mathrm{C}$ & 0 & 1 & 1 \\
\hline c.1519delT & 0 & 1 & 1 \\
\hline c. $1229 \mathrm{C}>\mathrm{T}$ & 0 & 1 & 1 \\
\hline c. $1340 \mathrm{~A}>\mathrm{T}$ & 0 & 1 & 1 \\
\hline \multicolumn{4}{|c|}{ Testing by 159 variant testing } \\
\hline c. $919-2 A>G$ & 5 & 14 & 19 \\
\hline c. $1229 \mathrm{C}>\mathrm{T}$ & 0 & 5 & 5 \\
\hline c. $2168 \mathrm{~A}>\mathrm{G}$ & 0 & 4 & 4 \\
\hline c. $1174 \mathrm{~A}>\mathrm{T}$ & 0 & 1 & 1 \\
\hline c. $1226 \mathrm{G}>\mathrm{A}$ & 0 & 1 & 1 \\
\hline c. $1336 \mathrm{C}>\mathrm{T}$ & 0 & 1 & 1 \\
\hline c. $1343 \mathrm{C}>\mathrm{A}$ & 0 & 1 & 1 \\
\hline c. $1343 \mathrm{C}>\mathrm{T}$ & 0 & 1 & 1 \\
\hline c. $1594 \mathrm{~A}>\mathrm{C}$ & 0 & 1 & 1 \\
\hline c.1692dupA & 0 & 1 & 1 \\
\hline c. $1707+5 \mathrm{G}>\mathrm{A}$ & 0 & 1 & 1 \\
\hline c. $589 \mathrm{G}>\mathrm{A}$ & 0 & 1 & 1 \\
\hline
\end{tabular}

Broader genetic testing can improve the diagnostic sensitivity and accuracy. By analyzing the patients who received 127 gene panel testing, we found that 25 patients had mutant genes which were not included in 159 variant testing, and nine of them carried pathogenic or likely pathogenic variants. Fifty-one patients carried variants that were not included in 159 variant testing, and thirty-six of them carried pathogenic or likely pathogenic variants. If these patients only received 159 variant testing, the genetic etiology would not be identified. This result revealed that even if the patients pass the traditional gene testing, they still could have genetic causes. Therefore, expanded gene testing should be done to identify the genetic etiology.

Moreover, accurate genetic testing can benefit patients in several aspects. Identifying genetic etiology can guide clinicians to determine treatment strategies. In our research, we found several patients carried variants in $\mathrm{CDH} 23$, WFS1, and PCDH15 by 127 gene panel testing. As reported in previous research, some patients with above gene mutation may have a poor prognosis of cochlear implantation (CI) [20]. Besides, early genetic diagnostic of hearing loss would provide more information on nature, mode of inheritance, and implications of genetic disorders, which would help individuals and families make informed medical and 
personal decisions [14, 21]. Furthermore, the relationship between genotype and phenotype of hereditary hearing loss is complicated; finding novel variants would broaden the understanding of hereditary hearing loss.

Genetic testing should be optimized according to multiple dimensions. Firstly, the common detection of deafness genes and variants should vary by ethnicity and region. For example, in 127 gene panel testing, c.109G>A was the most frequent variant, then was the c.235delC. In our research, we did not select the ethnicity of the patients, which would influence the frequency of these variants. A previous research in Qinghai (China) has shown that c.109G $>$ A was more common in minority patients, while c.235delC was more common in Han nationality [22]. Secondly, some gene mutation which might be associated with poor prognosis of CI such as POU3F4, TMPRSS3, PJVK, CDH23, and PCDH15 should be added in genetic testing. The full sequence of these genes or at least the exon regions should be detected. If pathological mutations in these genes are missed, it may bring catastrophic consequences to patients and their families.

\section{Conclusion}

Congenital deafness is the number one congenital disease that endangers human health. The identification of the cause through hearing screening combined with genetic testing is an important basis for mechanism research, clinical intervention, and genetic counseling. With the development of sequencing technology, deeper and broader genetic testing by next-generation sequencing are efficient and affordable. More accurate and convenient genetic testing should be designed and recommended. It can increase the detection rate of patients with hereditary deafness, guide clinical treatment strategies, expand new deafness genes or variants, and provide help for future clinical work.

\section{Data Availability}

The data which support the conclusions of our study is upon the request.

\section{Ethical Approval}

The study of the protocols was approved by the review boards of the ethics committees of the Tongji Medical College of Huazhong University of Science and Technology.

\section{Consent}

Written informed consent, clinical evaluations, and blood samples were obtained from all the participants or their legal guardians.

\section{Conflicts of Interest}

The authors declare that they have no conflict of interest.

\section{Authors' Contributions}

Le Xie and Yue Qiu contributed equally to this work.

\section{Acknowledgments}

This work was supported by a grant from the National Nature Science Foundation of China (81771003 and 82071058).

\section{Supplementary Materials}

See Supplementary Tables S1 and S2 in the Supplementary Material. Tested genes among 127 gene panel testing are provided in Table S1, and tested variants in 159 variant testing are provided in Table S2. (Supplementary Materials)

\section{References}

[1] R. Cabanillas, M. Diñeiro, G. A. Cifuentes et al., "Comprehensive genomic diagnosis of non-syndromic and syndromic hereditary hearing loss in Spanish patients," BMC Medical Genomics, vol. 11, no. 1, p. 58, 2018.

[2] A. M. H. Korver, R. J. H. Smith, G. van Camp et al., "Congenital hearing loss," Nature Reviews Disease Primers, vol. 3, no. 1, 2017.

[3] A. van Wieringen, A. Boudewyns, A. Sangen, J. Wouters, and C. Desloovere, "Unilateral congenital hearing loss in children: challenges and potentials," Hearing Research, vol. 372, pp. 2941, 2019.

[4] Y.-. B. Xiang, C.-. Y. Xu, Y.-. Z. Xu et al., "Next-generation sequencing identifies rare pathogenic and novel candidate variants in a cohort of Chinese patients with syndromic or nonsyndromic hearing loss," Molecular Genetics \& Genomic Medicine, vol. 8, no. 12, 2020.

[5] K. D. Brodie, A. T. Moore, A. M. Slavotinek et al., "Genetic testing leading to early identification of childhood ocular manifestations of usher syndrome," Laryngoscope, vol. 131, no. 6, 2020.

[6] A. E. Shearer, M. S. Hildebrand, and R. J. H. Smith, "Hereditary hearing loss and deafness overview, in GeneReviews( $\left.{ }^{\circledR}\right)$," in GeneReviews is a registered trademark of the University of Washington, Seattle, M. P. Adam, Ed., University of Washington, Seattle Copyright (C) 1993-2021, University of Washington, Seattle, Seattle (WA), 1993.

[7] D. K. Chan and K. W. Chang, "GJB2-associated hearing loss: systematic review of worldwide prevalence, genotype, and auditory phenotype," Laryngoscope, vol. 124, no. 2, pp. E34E53, 2014.

[8] F. F. Zhao, Y. B. Ji, D. Y. Wang et al., "Phenotype-genotype correlation in 295 Chinese deaf subjects with biallelic causative mutations in the GJB2 gene," Genetic Testing and Molecular Biomarkers, vol. 15, no. 9, pp. 619-625, 2011.

[9] G. Casazza and J. D. Meier, "Evaluation and management of syndromic congenital hearing loss," Current Opinion in Otolaryngology \& Head and Neck Surgery, vol. 25, no. 5, pp. 378-384, 2017.

[10] A. Eftekharian and M. H. Mahani, "Jervell and Lange-Nielsen syndrome in cochlear implanted patients: our experience and a review of literature," International Journal of Pediatric Otorhinolaryngology, vol. 79, no. 9, pp. 1544-1547, 2015.

[11] T. Yang, L. Guo, L. Wang, and X. Yu, "Diagnosis, intervention, and prevention of genetic hearing loss," Advances in Experimental Medicine and Biology, vol. 1130, pp. 73-92, 2019. 
[12] C. M. Sloan-Heggen, A. O. Bierer, A. E. Shearer et al., "Comprehensive genetic testing in the clinical evaluation of 1119 patients with hearing loss," Human Genetics, vol. 135, no. 4, pp. 441-450, 2016.

[13] T. Atik, G. Bademci, O. Diaz-Horta, S. H. Blanton, and M. Tekin, "Whole-exome sequencing and its impact in hereditary hearing loss," Genetics Research, vol. 97, 2015.

[14] G. García-García, A. Berzal-Serrano, P. García-Díaz et al., "Improving the management of patients with hearing loss by the implementation of an NGS panel in clinical practice," Genes, vol. 11, no. 12, 2020.

[15] Y. Sun, J. Xiang, Y. Liu et al., "Increased diagnostic yield by reanalysis of data from a hearing loss gene panel," $B M C$ Medical Genomics, vol. 12, no. 1, p. 76, 2019.

[16] S. Chen, Y. Jin, L. Xie et al., "A novel spontaneous mutation of the SOX10 gene associated with Waardenburg Syndrome Type II,” Neural Plasticity, vol. 2020, Article ID 9260807, 2020.

[17] Y. Qiu, S. Chen, K. X. Le Xie et al., "Auditory neuropathy spectrum disorder due to two novel compound heterozygous OTOF mutations in two Chinese families," Neural Plasticity, vol. 2019, Article ID 9765276, 2019.

[18] Y. Qiu, S. Chen, X. Wu et al., "Jervell and Lange-Nielsen syndrome due to a novel compound heterozygous KCNQ1 mutation in a Chinese family," Neural Plasticity, vol. 2020, Article ID 3569359, 2020.

[19] S. Chen, Z. Liang, B. Chen et al., "The prevalence of deafnessassociated mutations in neonates: a meta-analysis of clinical trials," International Journal of Pediatric Otorhinolaryngology, vol. 121, pp. 99-108, 2019.

[20] H. Zhang, S. Chen, Y. Sun, and W. Kong, "The value of genetic diagnosis of deafness in evaluating the prognosis of cochlear implantation," Lin Chung Er Bi Yan Hou Tou Jing Wai Ke Za Zhi, vol. 35, no. 3, pp. 274-281, 2021.

[21] N. B. Urbančič, S. Battelino, T. Tesovnik, and K. T. Podkrajšek, "The importance of early genetic diagnostics of hearing loss in children," Medicina, vol. 56, no. 9, p. 471, 2020.

[22] S. Duan, Y. Guo, X. Chen, and Y. Li, "Genetic mutations in patients with nonsyndromic hearing impairment of minority and Han Chinese ethnicities in Qinghai, China," Journal of International Medical Research, vol. 49, no. 4, 2021. 